Hope College

Hope College Digital Commons

Faculty Publications

7-2011

\title{
Compassionate reappraisal and emotion suppression as alternatives to offense-focused rumination: Implications for forgiveness and psychophysiological well-being
}

Charlotte vanOyen-Witvliet

HopeCollege,witvliet@hope.edu

Nathaniel J. DeYoung

Hope College

Alicia J. Hofelich

Hope College

Paul DeYoung

Hope College, deyoung@hope.edu

Follow this and additional works at: https://digitalcommons.hope.edu/faculty_publications

Part of the Physics Commons, and the Psychology Commons

\section{Recommended Citation}

Published in: Journal of Positive Psychology, Volume 6, Issue 4, July 1, 2011, pages 286-299. Copyright (c) 2011 Routledge Journal, Oxfordshire, England. The final published version is available at:

http://dx.doi.org/10.1080/17439760.2011.577091

This Article is brought to you for free and open access by Hope College Digital Commons. It has been accepted for inclusion in Faculty Publications by an authorized administrator of Hope College Digital Commons. For more information, please contact digitalcommons@hope.edu. 
Running head: OFFENSE RUMINATION, COMPASSIONATE REAPPRAISAL, and EMOTION SUPPRESSION

Compassionate reappraisal and emotion suppression as alternatives to offense-focused rumination:

\section{Implications for forgiveness and psychophysiological well-being}

Charlotte vanOyen Witvliet ${ }^{1}$, Nathaniel J. DeYoung ${ }^{\mathrm{a}}$, Alicia J. Hofelich ${ }^{\mathrm{a}}$, \& Paul A. DeYoung ${ }^{\mathrm{b}}$

a Psychology Department, Hope College, Holland, MI, USA; Physics Department, Hope College, Holland, MI, USA

${ }^{1}$ Corresponding author. Email: witvliet@hope.edu. 


\begin{abstract}
This within subjects experiment (28 females, 26 males) examined three responses to a past interpersonal offender. We contrasted offense-focused rumination with two subsequent, counterbalanced coping strategies: compassionate reappraisal and emotion suppression. Compassionate reappraisal emphasized the offender's human qualities and need for positive change. Emotion suppression inhibited the experience and expression of negative offense-related emotions. Offense rumination was associated with negative emotion, faster heartbeats (i.e., shortened ECG R-R intervals), and lower heart rate variability (i.e., the HF component of the RR power spectrum). By contrast, both compassionate reappraisal and emotion suppression decreased negative emotion in ratings and linguistic analyses, calmed eye muscle tension (orbicularis oculi $E M G$ ), and maintained heart rate variability at baseline levels. Suppression inhibited negative emotion expression at the brow (corrugator EMG) and slowed cardiac R-R intervals, but without forgiveness effects. Only compassionate reappraisal significantly increased positive emotions, smiling (zygomatic EMG), and social language along with forgiveness.
\end{abstract}

(148 words)

Keywords: compassion; forgiveness; reappraisal; suppression; forbear; rumination; facial EMG; heart rate variability (HRV); spectral analysis

Word count: 8462 plus Tables 


\section{Compassionate reappraisal and emotion suppression as alternatives to offense-focused rumination: Implications for forgiveness and psychophysiological well-being}

Positive psychology considers how people can promote happiness, well-being, and flourishing, even after negative events. In the wake of hurtful interpersonal transgressions, individuals may adopt a variety of responses to their pain, each of which has implications for well-being. In this psychophysiological experiment, we study three post-offense coping responses—rumination, reappraisal, and suppression— that are at the interface of the forgiveness literature (Witvliet \& McCullough, 2007) and the emotion regulation literature (Gross, 2007).

Research has identified that after an interpersonal offense, two distinct positive reappraisal strategies can effectively counter the negative emotions and physiological stress that characterize offense-focused rumination (Witvliet, Knoll, Hinman, and DeYoung, 2010). Regardless of whether reappraisals focused on benefits even in the difficult context of a transgression (e.g., insight, understanding, recognition of personal strengths) or on compassionate understanding of the offender's humanity and need for change, individuals calmed their negative self-reports, writing, and facial expressions (corrugator EMG), while increasing subjective happiness and positive emotion. Although the two positive reappraisals both down-regulated negative and up-regulated positive emotional responses, each had distinct effects. When using benefit-focused reappraisal, individuals generated the greatest increases in gratitude, joy, and smiling (zygomatic EMG), while significantly increasing the parasympathetic nervous system response of heart rate variability (i.e., the HF component of the R-R power spectrum). When using compassion-focused reappraisal, these same individuals cultivated the greatest increases in social, empathic, and forgiving responses while calming heartbeats (slowing 
of R-R intervals in the ECG) and arousal-linked tension in the face (orbicularis oculi EMG under the eye). The current study seeks to replicate compassion-focused reappraisal effects while widening its scope to test the suppression of offense-related negative emotion.

Theorists have proposed that some attempts to forgive may involve exerting self-control to quell negative emotional experiences and expressions—efforts that resemble emotion suppression (Witvliet \& McCullough, 2007). While the suppression of emotional expression has been well studied (see Gross, 2007), less attention has been given to the suppression of both inner emotional experiences and their outward expression in non-clinical participants (Dunn, Billotti, Murphy, \& Dalgleish, 2009). Furthermore, suppression of unforgiving emotions and expressions has not yet been experimentally examined (Worthington \& Sotoohi, 2009). This psychophysiological experiment is the first to address this lacuna in the literature by examining post-offense suppression, reappraisal, and rumination within individuals.

\section{Forgiveness Theory}

Forgiveness definitions vary in ways that relate to both reappraisal and suppression models. Despite their differences, researchers have some shared understandings about the nuances that characterize forgiveness (Worthington, 2005). These include distinguishing forgiveness from forgetting, condoning, justifying, excusing, exonerating, accepting, moving on, seeing justice served, and reconciling (see Enright, 2001). These distinctions are important for ensuring that forgiveness involves a clear understanding of the offender, the offense, and its implications. Distinguishing forgiveness from distorted interpretations of the offense, forgoing justice, and reconciliation is important for victim safety and justice. When forgiveness is fused with reconciliation and does not emphasize justice, victims may be at greater risk for experiencing subsequent transgressions from spouses (McNulty, 2010) or remaining with an 
abusive partner (Gordon, Burton, \& Porter, 2004). Victims’ clarity of self-concept and selfrespect may be adversely influenced when forgiving a perpetrator who has not indicated the victim is safe and valued (see Luchies, Finkel, McNulty, \& Kumashiro, 2010). It is important to support victims' differentiation of self, which serves as a mediator of the relationship between dispositional forgiveness and measures of spiritual stability, mental health, and positive emotion (Sandage \& Jankowski, 2010).

Forgiveness scholars in psychology also recognize that interpersonal forgiveness varies based on the nature of the offense and the relational context in which the transgression occurred (Maio, Thomas, Fincham, \& Carnelly, 2008). Even in close and long-term relationships such as families (mother-father-child), perceptions of whether forgiveness has been granted and received can differ, as can the factors that make forgiveness more or less likely (e.g., repeat offenses, apologies, forgiveness-related traits; Maio et al., 2008).

Although occurring in a relational context, states of interpersonal forgiveness involve a transformative process within the forgiver, even when reconciliation is not possible or wise. Sometimes forgiveness involves a decisional commitment, but it can also be experienced as emotional transformation (see Worthington, 2009 regarding decisional forgiveness and emotional forgiveness). Forgiveness may be characterized by reductions in negative unforgiving responses (e.g., less anger, avoidance, and revenge), and other times emotional forgiveness is one of supplanting negative emotions of unforgiveness with positive, prosocial responses that are benevolent (Witvliet \& McCullough, 2007; Worthington, 2005).

Scholars broadly agree that forgiveness involves processes and outcomes associated with reduced negative emotions, cognitions, motivations, and behaviors toward the offender (Worthington, 2005). In this sense, a person who self-restrains angry emotions, hateful thoughts, 
vengeful motivations, and retaliatory behaviors toward an offender may employ emotion suppression in the process of forbearing or forgiving. This study tests the emotional concomitants (physiology) and outcomes (ratings and writing) of suppressing the experience and expression of negative emotions while thinking about one’s offender.

Other conceptualizations of forgiveness that focus on emotional replacement of negative unforgiving responses with positive and prosocial responses note that this transformation may be either part of the process or a result of cultivating forgiveness (Worthington, 2005). The forgiveness literature has shown that empathy for the offender plays a pivotal role (see Enright, 2001; Enright \& Fitzgibbons, 2000; Hargrave, 2001; Worthington, 2009 for recent books on theory and application; see Subkoviak, Enright, Wu, \& Gassin, 1995 for scale development of the Enright Forgiveness Inventory; see McCullough, Worthington, \& Rachal, 1997; Witvliet, Ludwig, \& Vander Laan, 2001 for early empirical studies of empathy and forgiveness; see Sandage \& Worthington, 2010 for a recent intervention study of empathy-oriented forgiveness versus self-enhancing forgiveness, using the Enright Forgiveness Inventory). Experimental research has shown that the process of adopting a compassion-focused reappraisal of the offender as a human being who needs to experience positive transformation cultivated empathy and forgiveness characterized by both down-regulated negative responses and up-regulated positive emotions and a social orientation (Witvliet et al., 2010).

\section{The Current Study}

The current study brings together the forgiveness and emotion regulation literatures in an experimental comparison of compassionate reappraisal and suppression as self-restraint of one's negative emotions and expressions in responses to rumination about a past interpersonal offender and offense. This repeated measures experiment draws on established paradigms (e.g., 
Witvliet et al., 2001, 2010) to test the within subjects effectiveness of each coping strategy after offense rumination for promoting participants' forgiveness and their well-being. The effects of the two strategies are then compared to determine whether one strategy is more effective in countering the offense-related rumination responses. We assess well-being effects by 1) measuring subjective ratings of emotion, 2) conducting linguistic analyses of participants’ written descriptions of their thoughts, emotions, physical responses, and behavioral motivations, and 3) measuring physiological responses relevant to emotional expression and emotionregulation. These include measures of even subtle displays of emotion on the face detected with electromyographic (EMG) measures at the cheek (zygomatic) associated with positive emotion, at the brow (corrugator) associated with negative emotion, and under the eye (orbicularis oculi) associated with aroused emotions (Witvliet \& Vrana, 1995). We also assess cardiac measures because of relationships between emotion regulation and cardiac functioning, (e.g., Mauss, Cook, Cheng, \& Gross, 2007; Witvliet et al., 2010). In particular, we assessed cardiac reactivity by measuring the time between heartbeats (R-R intervals in the ECG waveform). We also measured heart rate variability (HRV) because it is as an indicator of regulatory control via the parasympathetic nervous system, the system which calms the body's aroused "fight-or-flight" response (e.g., Thayer \& Brosschot, 2005). We now turn to examine the literature pertinent to the three experimental conditions of offense-focused rumination and both coping strategies: compassionate reappraisal and negative emotion suppression.

\section{Offense-Focused Rumination}

Repetitive thinking about a past interpersonal offense has been found to activate negative emotions and thwart empathy and forgiveness within victims (Witvliet et al., 2001, 2008, 2010). Longitudinal increases in offense-focused rumination were associated with impaired forgiving, 
and this was mediated by anger toward the transgressor (McCullough, Bono, \& Root, 2007). More frequent transgression ruminations were associated with less forgiveness of a spouse toward whom they held a combination of positive and negative feelings (Kachadourian, Fincham, \& Davila, 2005).

Post-offense repetitive thinking may have both adaptive and maladaptive functions. In light of Watkins' (2008) conceptualization, it can be an attempt to understand or resolve meaning-based or goal-based discrepancies (e.g., between the way things were before the offense and now, and between how they are now and how they ought to be). Victims may engage in offense-focused rumination in an effort to understand what happened and with the goal of preventing future relational harm. Outside the transgression context, other types of repetitive thought or rumination can be adaptive, particularly when action-focused (Ciarocco, Vohs, Baumeister, 2010) on maintaining a goal (Atamirano, Miyake, \& Whitmer, 2010).

However, repetitive thinking about the negatively valent offense and its implications is likely to yield some adverse outcomes for victims (see Watkins, 2008). Post-offense rumination has been associated with impaired HRV (Witvliet et al., 2010), increased blood pressure and sweat (skin conductance) levels (Witvliet et al., 2001), as well as tension under the eye (orbicularis oculi), and tension at the brow muscle (corrugator) associated with negative emotion (Witvliet et al., 2001, 2010). More broadly, ruminative reviews of one’s problems and emotions is associated with impaired problem-solving, depression, lower motivation, and decreased social support (Nolen-Hoeksema, Wisco, \& Lyubomirsky, 2008). As a response style, rumination has been strongly correlated with psychopathology, including anxiety disorders, depression, eating disorders, and substance disorders (Aldao, Nolen-Hoeksema, \& Schweizer, 2010). 


\section{Reappraisal}

Cognitive reappraisal is understood as a process of interpreting an emotional circumstance in ways that can change the situation's emotional impact, often by down-regulating negative emotions (see Gross, 1998), and also by up-regulating positive emotions (Gross 2007; Rottenberg \& Gross, 2007). As such, reappraisal has been considered a constructive repetitive thought approach (Watkins, 2008) and an effective emotion repair and regulation strategy (Augustine \& Hemenover, 2008). As a response style, reappraisal has had a small to moderate inverse relationship to psychopathology, and was associated with lower levels of depression and anxiety symptoms (Aldao et al., 2010). Reappraisal has been considered a healthy approach to emotion regulation that is further associated with more positive emotions and relationships, life satisfaction, self-esteem, optimism, and overall well-being (Gross \& John, 2003).

Compassion-focused reappraisal emphasizes the complex humanity of the offender and interprets the offense as evidence that the offender needs to experience positive growth or transformation (Witvliet et al., 2010). This emphasis on the offender as a human being (rather than totalizing the offender as a liar, cheat, thief, or betrayer) and on the ways the offender needs to grow or change has the capacity to help victims find a genuine way to wish the offender well, although it can be difficult to cultivate (see Enright, 2001; Enright \& Fitzgibbons, 2000; Sandage \& Worthington, 2010). Research with crime victims, their family, and friends found that those who valued warmth-based virtues such as compassion and love even more than conscientiousness-based virtues such as self-control and justice were more likely to grant forgiveness to the offender (Berry, Worthington, Wade, Witvliet, \& Kiefer, 2005). In Worthington's (2009) frame, this can be described as responsible compassion and forgiveness 
without excusing injustice, a vital distinction that is needed to protect victims (see Gordon et al., 2004; Luchies et al., 2010; McNulty, 2010).

When faced with difficult situations, reappraisers tend to overcome anger and show more adaptive cardiovascular responses (Mauss et al., 2007). Experimentally, reappraisal versus rumination about an upsetting event was associated with less anger, cognitive perseveration, and sympathetic nervous system activity (Ray, Wilhem, \& Gross, 2008). Compassionate reappraisal in particular stimulated empathy and forgiveness along with increased positive emotion, reduced negative and aroused emotion (including anger), decreased facial EMG at the brow (corrugator) and under the eye (orbicularis oculi), and slowed cardiac R-R intervals in comparison to offensefocused rumination (Witvliet et al., 2010). This was consistent with other research associating forgiveness-as opposed to offense rumination — with less sympathetic nervous system input to the heart (measured with rate pressure products; Lawler et al., 2003; Lawler, Karremans, Scott, Edlis, \& Edwards, 2008; Witvliet et al., 2008), lower blood pressure (Friedberg, Suchday, \& Shelov, 2007) and heart rate (Witvliet et al., 2001), reduced myocardial perfusion defects during anger-recall (Waltman et al., 2009), and fewer reported illness-related symptoms (Bono, McCullough, \& Root, 2008). The current study seeks to replicate compassionate reappraisal effects while comparing reappraisal to a self-control strategy that suppresses the experience and expression of negative emotion while thinking about one's offender and offense.

\section{Suppression}

Emotional suppression involves processing an emotion-evoking stimulus while inhibiting emotional experiences and expressions. The suppression literature focuses primarily on negative emotion expressions. Suppressing outward expressions of emotion while viewing negative emotion stimuli such as pictures or films is often associated with adverse effects (see Gross, 
2007), such as increased sympathetic nervous system responding (Gross \& Levenson, 1993, 1997), higher blood pressure in suppressors and their conversation partners, reduced memory formation (Butler et al., 2003), and declines in incidental memory for information presented during emotionally suppressive behavior (Richards \& Gross, 1999). Suppression of expression may further decrease positive emotion, deplete cognitive resources, and reduce social support (see Gross \& John, 2003). Suppressive coping is also associated with adverse health effects (see Giese-Davis \& Spiegel, 2003; Ryff \& Singer, 2003), and it has a moderate to large association with psychopathology, including anxiety, depression, and eating disorders (Aldao et al., 2010). Suppression may, however, be associated with some adaptive features (Dunn, et al., 2009). In addition to restraining aggressive behaviors (see Gross \& Levenson, 1997), Gross and Levenson (1993) found that suppressing expressions during a disgusting film was associated with decreased fear reports and heart rate. It may be that suppression operates more effectively in participants without high levels of negative affect. Whereas Dalgleish, Yiend, Schweizer, \& Dunn (2009) found that high negative affect participants experienced increases in negative emotion after suppressing their emotions for a negative life event, Dunn et al. (2009) found that healthy participants showed a more adaptive suppression effect. Wegner, Erber, and Sanakos (1993) found that participants in their experiment could successfully down-regulate their negative mood (suppress) except when they were under added cognitive load.

Another approach to suppression includes intentional attempts to thwart both the internal experience and external expression of negative emotion. Dunn et al. (2009) emphasize that for many individuals who are coping in their daily lives, the goal is to regulate both their internal emotions and their external expressions. This dual emphasis approach associates suppression with resilience. Dunn et al. (2009) found that when healthy participants suppressed internal 
feelings and external expressions during a traumatic film, they experienced less fear and had heart rate and electrodermal activity equivalent to the control group. At one week delay, suppressors had lower intrusive imagery and free recall of the traumatic film content, even though their recognition memory for the traumatic film material was intact. Dunn et al. (2009) argued for a more nuanced view of suppression that includes down-regulating internal experiences and outward expressions of unwanted emotion in non-clinical samples.

\section{Experiment Hypotheses}

The compassionate focus of our reappraisal condition bears similarity to the approaches of Enright (2001), Hargrave (2001), Sandage \& Worthington (2010), Worthington (2009), and Witvliet et al. (2001, 2010). Thus, we measure ratings of empathy and forgiveness as manipulation checks. Based on the literature, we also predicted that compassionate reappraisal would prompt the most positive emotion ratings in the experiment while also diminishing negative emotion ratings (see Witvliet et al., 2001, 2010). In linguistic analyses of participants' writing, we expected compassionate reappraisal to yield higher word counts related to forgiveness and positive emotion, and lower negative emotion word counts. Because the explicit focus of compassion is beyond oneself and on the offender, we predicted that participants would write with more social references than when they suppressed their own emotional experiences and expression. We also used indirect narrative assessments that do not count words but rather test for the closeness of concept representations in analyses that mimic human knowledge representations. We predicted that compassionate reappraisal would yield narratives more closely aligned with a forgiveness text probe and a positive emotion text probe, and more distant from a negative emotion text probe. Consistent with emotion predictions and past research (Witvliet et al., 2001, 2010), we hypothesized that compassionate reappraisal would be 
associated with less tension at the brow (corrugator) and under the eye (orbicularis oculi) and that heartbeats (R-R intervals) would be slower than during offense rumination. One prior study examined parasympathetic nervous system activity related to rumination and compassion. Witvliet et al. (2010) found that regulatory control via the parasympathetic nervous system (measured with HRV) decreased significantly from pretrial baseline to offense rumination. By contrast, compassion-focused reappraisal was associated with parasympathetic activity (using HRV) that was statistically equal to pretrial baseline relaxation periods. Thus, we predicted similar patterns in the present study.

This is the first study to compare reappraisal to the suppression of emotional experiences and expressions during transgression-related thoughts. Dunn et al. (2009) have described the maladaptive suppression hypothesis and the adaptive suppression hypothesis, which are related to research methodology. Our method is more aligned with Dunn et al. (2009) in studying emotion suppression in a nonclinical sample with the instructions to inhibit emotional experiences and expressions while focusing on the emotional content of the condition. We predicted that compared to rumination, participants would produce less negative emotion ratings and written narratives while suppressing their negative emotional experiences and expressions. Gross and Levenson (1993) found that suppression reduced facial expressions and heart rate despite measures suggesting heightened sympathetic nervous system activity. We predicted that compared to rumination's aroused and negative physiology effects, suppression would subdue facial expressions and heart rate. However, we held these predictions tentatively in light of the exploratory nature of post-offense emotion suppression. 


\section{Methods}

\section{Participants}

Fifty-four introductory psychology students (26 males, 28 females) completed the experiment as one way to meet a research requirement. The average age of participants was 18.78 years $(S D=$ 0.69). Participants in this study did not specify ethnicity. However, in similar studies at this location, approximately $85-90 \%$ of participants self-identified as White, with between $1 \%$ and 4\% self-identifying with particular ethnic identities: African-American, Latino, Asian-American, and others. Participants reported transgressions that were committed primarily by romantic partners (40\%) and friends (28\%), occurred an average of 15 months prior to the study, and were rated as $4.65(S D=1.56)$ in severity ( 1 Not at all distressing -7 Extremely distressing).

\section{Design}

We used a repeated measures within-subjects experimental design similar to that used by Witvliet et al. (2010). In a 90-minute paradigm, the participant completed four trials. Each trial was comprised of a 120s pre-trial baseline followed by 120s period in which the participant ruminated about the past real-life offense or implemented a coping strategy. Each pretrial baseline served to physically relax and clear the participant's mind by mentally repeating a nonemotional word (Witvliet \& Vrana, 1995, Witvliet et al., 2001, 2010) before the subsequent offense-related rumination or coping induction. The pretrial baseline was also important for determining the physiological effects of each experimental condition; we calculated changes that occurred as participants went from pretrial baseline to imagery (i.e., rumination or coping). We did not measure self-reports after pretrial baseline periods because this would induce movementrelated physiological reactivity that would decrease the accuracy of experimental condition effects. 
Half of the participants (blocked by gender) were randomly assigned to proceed from offense rumination to compassionate reappraisal and then from offense rumination to emotion suppression. The other half were assigned to proceed from offense rumination to emotion suppression and then from offense rumination to compassionate reappraisal.

\section{Procedure}

Participants gave informed consent and were tested individually in the laboratory. As they sat in a recliner in front of a flat screen monitor, the electrodes and physiological recording devices were affixed and monitored in the adjacent equipment room. The electrode areas were prepared using standard methods (Witvliet \& Vrana, 199; Witvliet et al., 2010). All physiological measures were tested for clear and reliable signals before beginning measurement.

Next, each participant completed a questionnaire to identify and describe a particular prior offense in which another person hurt and offended him or her. This particular interpersonal offense became the basis for all subsequent experimental conditions in the study (described in detail below). When all imagery periods and accompanying measures were completed, physiological recording devices were removed, and participants were debriefed.

\section{Stimulus materials}

Instructions were displayed for 30-seconds on a computer monitor directly in front of the participant. A tone signaled participants to open their eyes and read the relevant pre-trial baseline relaxation or imagery instructions for offense-related rumination, compassionate reappraisal, or emotion suppression. Experimental instructions directed participants to close their eyes for all baseline and imagery periods. 


\section{Pre-trial baseline instructions}

"Quietly rest in your chair at this time. Your job is to sit, relax and think the word 'one' when you naturally breathe out. Keep your arms, legs, and body still as you remain quiet for two minutes, thinking 'one' as you sit here.”

\section{Offense rumination imagery instructions}

“Think about the person who hurt you and all the ways that this offense was hurtful to you. For the next two minutes, remember what happened and the thoughts, feelings, and physical reactions you had. What did you think? How did you feel? How did your body react? What did you do? Think of all the ways that you were affected by the hurtful experience.”

\section{Compassionate reappraisal instructions}

"Now your job is to re-think your response to the offender. For the next two minutes, think of the offender as a human being who behaved badly. Even if the relationship cannot be restored, try to genuinely wish that this person experiences something positive or healing. Even though it may be hard, focus your thoughts and feelings on giving a gift of mercy or compassion.”

\section{Emotional suppression instructions}

"Your job right now is to think about the offender and the offense. At the same time, try not to become emotional about the experience, and try not to show any outward expression of feelings you might have. For the next two minutes, think about the offender and the offense, but if you notice any upset or negative emotions, try not to feel or show them.”

\section{Self-Reports}

\section{Ratings}

Participants privately recorded their ratings after completing each offense-related rumination and coping condition. SuperLab software randomly ordered questions about participants' subjective 
emotions and experiences. Using a Biopac RB-730 response-pad with a seven-point scale, participants rated their emotional valence ( 1 = negative to 7 = very positive), perceived control ( $1=$ not in control to $7=$ very much in control), and the degree to which they experienced anger, anxiety, sadness, empathy, a commitment to forgive the offender, and heartfelt forgiveness toward the offender $(1=$ not at all to 7 = completely). The latter two ratings were single-item measures developed with Worthington (2009) to assess concepts of decisional forgiveness (commitment to forgive) and emotional forgiveness (heartfelt forgiveness). Based on the work of Witvliet et al. (2010), the empathy and forgiveness ratings were used to assess the manipulation of compassion in comparison to rumination.

Analyses of written responses: Latent Semantic Analysis (LSA) and Linguistic Inquiry and Word Count (LIWC)

After each rumination and coping condition, participants were prompted to describe their experience during imagery by typing sentences in response to four questions on a laptop: "What were you THINKING during your imagery? What were you FEELING during your imagery? What were your PHYSICAL REACTIONS during your imagery? What do you WANT TO DO or SAY to your offender?” Latent semantic analysis (LSA: http://lsa.colorado.edu) was used to compare the narrative a participant produced in an experimental condition to a comparison forgiveness text, a positive emotion text, and a negative emotion text. ${ }^{1}$

For word-count assessments of participants' rumination and coping responses, Linguistic Inquiry and Word Count (LIWC: Pennebaker, Booth, \& Francis, 2007) software counted the number of words in submitted texts that matched dictionaries. The standard LIWC English dictionary containing the social language category was used to analyze the paragraphs from each experimental condition. We used the Witvliet et al. (2010) forgiveness dictionary, which detects 
word stems (altruism, amend*, compassion*, empath*, forgave, forgiv*, love*, loves*, loving*, merciful*, mercy*, sympath*). We also tested a shorter forgiveness dictionary based on the LSA forgiveness probe (forgive*, forgiveness*, compassion*, merciful*, mercy*). Statistical significance patterns were the same regardless of which forgiveness dictionary we tested. We report results for the Witvliet et al. (2010) dictionary.

\section{Physiology $y^{2}$}

During the 120s pretrial baseline and 120s imagery periods for each of the four conditions, we continuously measured physiological responses using standard methods (Biopac MP150 and Acqknowledge 3.9 software for an Apple iMac G5). We measured covert facial muscle activity relevant to emotion using standard electromyography methods at the zygomaticus (cheek) muscle linked to positive valence, the orbicularis oculi (under eye) muscle associated with affective arousal, and the corrugator supercilii (brow) muscle linked with negative valence (Witvliet \& Vrana, 1995).

We used electrocardiography (ECG) to obtain R-R (beat-to-beat) interval data. We then assessed heart rate variability (HRV) because it is an indicator of parasympathetic nervous system activity important in emotion regulation. HRV was calculated using a frequency domain method derived from R-R (beat-to-beat) intervals from the ECG data collected during each pretrial baseline and subsequent imagery period for each of the experimental conditions. ECG waveforms and R-R plots were evaluated for accuracy. All Power Spectrum Density (PSD) plots were inspected for a well-defined High Frequency (HF) peak. Finally, we reviewed correlational plots of HF values and root mean square successive difference (RMSSD) calculations based on the R-R data to identify possible outliers in each baseline and imagery period. An integral of the 
0.15-0.4 Hz HF range of the PSD was used as the heart rate variability measure indicative of the parasympathetic influences on the cardiac cycle (Task Force, 1996). ${ }^{3}$

The physiological data for each condition were calculated as change from each condition's pretrial baseline to the subsequent imagery period. For facial EMG, we used standard methods (Witvliet \& Vrana, 1995; Witvliet et al., 2001, 2010) to subtract the value for the final 10-sec epoch of each condition's pretrial baseline from each 10-sec epoch during imagery and then analyzed the change averaged across imagery epochs. Heart rate variability (HRV) was calculated based on the full 120s period because calculations of HRV are time dependent and require comparisons of equal durations (Task Force, 1996). Consistent with HRV methods, we calculated R-R (beat-to-beat) interval data for the full 120s period because these values are collected on a beat-by-beat basis. HRV and R-R data were analyzed based on change from the 120s pretrial baseline to the subsequent 120s imagery for each rumination and coping condition.

\section{Statistical analyses}

All ratings, written narratives, and physiological data in this repeated measures, within-subjects design were analyzed using repeated measures multivariate analyses of variance ANOVA (Witvliet et al., 2010). Means, $F$ values, $p$ values, and partial $\eta^{2}$ statistics are reported in the tables. Table 1 presents the self-report means and statistical effects for compassionate reappraisal compared to its relevant offense rumination and emotion suppression compared to its relevant offense rumination. Note that all self-report data were measured immediately after the imagery (i.e., rumination, coping) trial ended, whereas all physiological data were measured simultaneously during imagery and represent a change from the pretrial baseline for each condition. Table 2 reports results from a comparison of the effects of the two coping strategies. For this, values for compassionate reappraisal minus its preceding rumination trial were 
statistically compared to the values for emotion suppression minus its preceding rumination trial.

\section{Results}

\section{Self-reports}

[Tables 1 and 2 near here]

Ratings

Supporting the compassionate reappraisal manipulation, this condition was associated with significantly higher empathy, decisional forgiveness, and emotional forgiveness than offense rumination (see Table 1). Emotional suppression had no effect on forgiveness ratings, but it was associated with higher empathy than rumination. As Table 2 shows, the effect of compassionate reappraisal was significantly greater than the effect of emotion suppression for increasing empathy and both forgiveness ratings.

Compared to their respective offense rumination conditions, both coping strategies significantly increased ratings for emotional valence, and significantly decreased ratings of anger, anxiety, and sadness (see Table 1). However, only compassionate reappraisal moved valence ratings to the positive side of the scale and significantly increased perceived control. Table 2 further shows that the compassionate reappraisal effect of increasing emotional valence (negative - positive) from rumination levels was significantly greater than the effect of emotion suppression.

Linguistic analyses

As shown in Table 1, Latent Semantic Analysis (LSA) with the negative emotion word probe (Cronbach's alpha $=0.79)$ revealed that both compassionate reappraisal and emotion suppression narratives were more distant from the negative affective probe than narratives for the 
corresponding rumination trials. Table 2 shows that the change induced by both coping strategies was the same. Tests with the positive emotion text probe (Cronbach's alpha $=0.62$ ) revealed that only the compassionate reappraisal was closer to the positive language probe compared to rumination. Tests with the forgiveness text probe (Cronbach’s alpha $=.60$ ) showed that rumination and compassionate reappraisal were equally close to the forgiveness-related language representation, but emotion suppression was associated with narratives that were distant from the forgiveness probe.

Linguistic Inquiry and Word Count (LIWC) analyses revealed that in comparison to the relevant offense rumination, both compassionate reappraisal and emotion suppression equally decreased negative emotion words. Both also increased positive emotion words; however, Table 2 shows that this positive word increase was significantly greater for compassionate reappraisal compared to suppression. Only compassionate reappraisal increased social language compared to the preceding offense condition, replicating prior research (Witvliet et al., 2010). Compassionate reappraisal also increased forgiveness word use more than offense rumination, whereas emotion suppression did not increase forgiveness language, whether assessed with the LSA forgiveness probe words or with the forgiveness dictionary of Witvliet et al. (2010).

\section{Physiology}

Facial electromyography (EMG)

Rumination was associated with higher orbicularis oculi EMG reactivity levels than compassionate reappraisal, which is consistent with past research on empathic perspectivetaking, forgiveness that finds even a small way to wish the offender well (Witvliet et al., 2001), and compassion-focused reappraisal (Witvliet et al., 2010). We had hypothesized that corrugator EMG reactivity levels at the brow would also be lower during compassionate reappraisal than 
rumination. Although the relationship of means was consistent with the hypothesis, the difference was not statistically significant.

Emotion suppression had significantly lower orbicularis oculi and corrugator EMG reactivity levels and marginally lower zygomatic EMG levels relative to offense rumination. This overall pattern is consistent with the emotion suppression instruction not to feel or show negative emotions, which may have decreased facial emotion displays generally.

As shown in Table 2 the effects of the two coping strategies were significantly different only for the zygomatic muscle. Whereas compassionate reappraisal increased zygomatic (smiling) activity compared to rumination, suppression decreased smiling relative to rumination.

\section{Cardiovascular measures}

Only emotion suppression significantly slowed R-R intervals (i.e., similar to slower heart rate) compared to offense rumination. We had predicted that compassionate reappraisal would also produce longer cardiac R-R intervals than rumination, but this did not occur. To determine whether we replicated Witvliet et al.'s (2010) results, we conducted post-hoc analyses of pretrial baseline versus imagery values for each experimental condition. Replicating past research, we found that offense rumination accelerated R-R (beat-to-beat) intervals in the ECG waveform compared to pretrial baseline, $F(1,44)=4.47, p \leq .05$, partial $\eta^{2}=.09$. Compassionate reappraisal R-R intervals were statistically equivalent to pretrial baseline relaxation levels, $F$ (1, 45) $=0.11, p=.74$, partial $\eta^{2}=.002$. Suppression slightly slowed R-R intervals, but not significantly compared to pretrial baseline $F(1,43)=3.11, p=.085$, partial $\eta^{2}=.07$.

While compassionate reappraisal was associated with better parasympathetic functioning (i.e., higher HRV results for the HF component of the R-R power spectrum) compared to rumination, the effect was not significant (see Witvliet et al., 2010). Suppression and rumination 
HRV values did not differ statistically. Replicating past research (Witvliet et al., 2010), rumination impaired HRV compared to pretrial baseline levels, $F(1,39)=6.38, p \leq .05$, partial $\eta^{2}=.14$. Also consistent with Witvliet et al.'s (2010) compassion data, each coping strategy was

statistically equivalent to pretrial baseline relaxation levels (both $F \mathrm{~s}<1.22, p \mathrm{~s}>.28$, partial $\eta^{2} \leq$ $.03)$.

\section{Discussion}

This experiment investigated forgiveness and psychophysiological well-being in the context of coping with a real-life interpersonal offense. We were particularly interested in understanding constructive and unconstructive repetitive thoughts (see Watkins, 2008) and emotion-regulation strategies (see Gross, 2007) in the post-transgression context. Overall, we found points of convergence with the forgiveness-related literature. Rumination about the hurtful transgression and its implications increased negative emotional experiences and expressions, linguistically and physiologically, relative to other coping strategies. In this non-clinical young adult college sample, both a compassionate reappraisal strategy and a self-restraining emotion suppression strategy reliably decreased these negative emotion experiences and expressions. However, only compassionate reappraisal generated positive emotion, as measured with subjective ratings, analyses of written narratives, and facial muscle activity linked to smiling (zygomatic EMG). The findings are consistent with the forgiveness and emotion-regulation literatures, and we consider implications for victim flourishing.

\section{Offense rumination contributions}

Repetitively thinking about the hurtful offense and its implications was associated with negative emotion ratings and writing, more intense negative emotion expressions on the face, an 
accelerated cardiac cycle (i.e., shorter R-R intervals), and impairment of the parasympathetic response (i.e., HRV as the HF component of the R-R power spectrum). These findings replicate research linking offense rumination with negative emotion appraisals and facial expressions, faster heart rate (Witvliet et al., 2001), and decreased HRV (Witvliet et al., 2010). Such cardiovascular reactions, especially when heightened and sustained in vulnerable populations, can be associated with cardiovascular disease and increased mortality (see Task Force, 1996).

Post-offense rumination in the short term was associated with sadness, anxiety, and anger, along with negative emotion expressions, consistent with other experimental studies (Witvliet et al., 2001, 2008, 2010). Watkins (2008) identified that classes of repetitive thought which focus on negative content in an adverse context and in abstract ways are more likely to be destructive than adaptive. While the current study found immediate effects, rumination has been found to predict increases in depression symptoms over days, weeks, months, and years (see Aldao et al., 2010). As a response style, the tendency to negatively ruminate has a strong, positive association with psychopathology, showing particular relationships with symptoms of anxiety, depression, eating disorders, and substance disorders (Aldao et al., 2010). Given these immediate and long-term risks of rumination, it is important to understand the effects of alternative approaches people may use in attempts to cope with the offense and its implications.

\section{Coping strategies}

When coping with a hurtful interpersonal offense, what are the effects of strategies that attempt to suppress or reappraise? Suppression of emotion requires self-control as one inhibits or forbears negative responses toward an offender (see Worthington \& Sotoohi, 2009). Compassionate reappraisal views the offender as a complex human with limitations and flaws (e.g., past hurts, immaturity, undeveloped virtue, and/or psychopathology) and uses that insight 
to cultivate mercy. This perspective may aid in genuinely desiring that person's growth, learning, healing, and/or transformation (Witvliet et al., 2010), even if reconciliation does not occur. Compassion can navigate between the extremes of devaluing and idealizing the offender, and it plays a role in several forgiveness theories and applications (see Enright, 2001; Enright \& Fitzgibbons, 2000; Hargrave, 2001; Sandage \& Worthington, 2010; Worthington, 2009). We found that both emotion suppression and compassionate reappraisal resisted the negative patterns associated with offense rumination. Both strategies were associated with calm cardiac responses (i.e., slower R-R intervals) and stronger parasympathetic nervous system responses (i.e., higher HRV scores) that were statistically equal to pretrial baseline relaxation levels, unlike rumination which significantly accelerated heartbeats and significantly impaired HRV compared to pretrial baseline. The differences between these coping strategies highlight distinctions in the processes employed, in forgiveness findings, and in positive relational elements of well-being and flourishing.

\section{Suppression contributions}

When people were instructed in the suppression condition to inhibit their offense-related feelings and not express them, they succeeded in doing so. These results are consistent with Dunn et al.'s (2009) adaptive suppression hypothesis. Participants rated their emotions as more moderate in valence, wrote about their coping experience less negatively, and showed less of the negative facial expressions aroused by rumination. They also had calmer cardiac responses (R-R intervals). This may be because they indeed quelled their inner emotional feelings about the offense and/or because by subduing their negative expressions, the heart did not have to work as hard to recruit resources for emotion displays (Gross \& Levenson, 1993). 
Emotion suppression was associated with immediate negative reinforcement through the alleviation of negative emotion and responses. It is interesting to note that the term "suppression" generally holds negative connotations, whereas forbearance-which Worthington and Sotoohi (2009) conceptualize as suppression—has more positive connotations. Forbearance and suppression may effectively down-regulate negative emotion in the short term for less than traumatic offenses, and in nonclinical populations who are not experiencing high negative affect (Dalgleish et al., 2009) or cognitive load (Wegner et al., 1993). We are concerned, however, that the short term negative reinforcement of emotion suppression may have a longitudinal cost. Aldao et al. (2010) observed that suppression is associated with an increase in depressive symptoms over weeks and months. A suppressive coping style also had a moderate to large relationship to psychopathology generally, and particularly for anxiety, depression, and eating disorders.

In terms of promoting forgiveness, the current data show that emotion suppression is ineffective. Although emotion suppression was associated with higher empathy ratings than rumination was, this increase was significantly smaller than the empathy increase prompted by compassionate reappraisal. In fact, linguistic analyses with LSA showed that suppression moved people away from using forgiveness-relevant language in an assessment that mimics human knowledge representations. Word count analyses with LIWC showed that suppression and rumination prompted the same number of forgiveness references, and both were significantly lower than compassionate reappraisal. Suppression also failed to increase participants' ratings of decisional forgiveness and emotional forgiveness, whereas compassionate reappraisal increased both forgiveness ratings. In Worthington and Sotoohi's (2009) framework, use of forbearance 
may restrain negative emotions and expressions in the short term, but it does not resolve or transform offense-related negative responses.

\section{Compassion contributions}

Compassionate reappraisal offered a more transformative experience within the individual that cultivated increased positive and prosocial responses and decreased negative responses across ratings, linguistic analyses, and physiology. This pattern is consistent with forgiveness theory (Enright, 2001; Hargrave, 2001; Worthington, 2009). Supporting the manipulation, compassionate reappraisal was the more empathic response that cultivated significant increases in decisional and emotional forgiveness ratings, and in forgiveness word usage in written narratives. Compassionate reappraisal also uniquely increased the use of social words, positive ratings and word use, and activity at the zygomatic muscle associated with smiling expressions.

Given that compassionate reappraisal reduced negative emotion and increased positive emotion effects consistent with hypotheses, we were surprised that compassionate reappraisal did not significantly subdue corrugator EMG and calm the cardiac cycle compared to rumination. However, the means for both variables were in the predicted direction. We propose that this may be due to a smaller sample size in the current study (cf. Witvliet et al., 2010), and to a key difference in the compassionate reappraisal instructions. Unlike Witvliet et al.’s (2010) study, the current instructions included an acknowledgement that cultivating compassion for the offender may be difficult. While this acknowledgement of difficulty could be viewed as validating a client's efforts in a therapeutic setting, in the laboratory encounter these words may have primed participants to experience the task as difficult, resulting in a less positive reappraisal.

In keeping with the current experiment's immediate emotion regulation benefits, reappraisal as a response style is associated with lower levels of anxiety and depression 
symptoms (Aldao et al., 2010). Still, meta-analytic results show that reappraisal's beneficial relationship to mental health is small, whereas suppression has a medium to large association and rumination has large magnitude associations with psychopathology (Aldao et al., 2010).

While compassion has many positive associations, some individuals and relational contexts may carry risks. We echo theorists who emphasize the importance of maturity and selfdifferentiation that allows victims to resist polarized responses that devalue or over-idealize the offender (see Sandage \& Jankowski, 2010). Compassion needs to be considered along with power and justice concerns so that forgiveness does not place persons at risk in terms of selfconcept clarity or self-respect (Luchies et al., 2010), especially when transgressors could generate additional harm (see Gordon, Burton, \& Porter, 2004; McNulty, 2010).

\section{Summary and Conclusions}

Emotion regulation evidence from the current study suggests that if one's goal is only to downregulate negative emotional experiences in the short term, both suppression and compassionate reappraisal have equivalent effects. However, if an individual has the goal of forgiving an offender, then compassionate reappraisal is a forgiveness strategy that increases positive emotions and social responses. These changes were found with only one induction of each offense-related response, suggesting that these college students were experiencing the beginnings of transformation. In everyday coping, we suspect that people are likely to more often attempt to down-regulate negative emotion through suppression than develop compassionate reappraisal. If so, prior practice of suppression strategies may have yielded significant benefits immediately in the laboratory. Additional research is needed to determine the personal and situational conditions under which compassion may promote a just forgiveness that promotes responsible flourishing for individuals and relationships over time (Worthington, 2009). 


\section{Acknowledgements}

We gratefully acknowledge the support offered through a grant to the first author from the Fetzer Institute. The work of the second author was supported in part by a grant to Hope College from the Howard Hughes Medical Institute through the Undergraduate Science Education Program. This work is part and product of a project on The Pursuit of Happiness in Interdisciplinary Perspective directed by the Center for the Study of Law and Religion at Emory University and supported by a grant from the John Templeton Foundation. The views expressed do not necessarily reflect the views of the Center for the Study of Law and Religion or The John Templeton Foundation. We thank Heath Demaree for consultation on the experimental paradigm and heart rate variability; Christopher Barney for consultation on cardiovascular variables; Terry Blumenthal on facial EMG; Al Dueck on Latent Semantic Analysis; and John Shaughnessy for consultation on analyses. 


\title{
Notes
}

\begin{abstract}
${ }^{1}$ Latent semantic analysis (LSA: http://lsa.colorado.edu) simulates representations of human knowledge. LSA uses a semantic corpus based on a large repertoire of Western writing from the third grade level through the first year of college and then applies a technique similar to factor analysis (singular value decomposition). Within the semantic space, LSA determines the similarity of two texts by calculating a cosine value. Here, we compared the narrative a participant produced in an experimental condition to three types of "probes": a comparison positive emotion text, a negative emotion text, and a forgiveness word text. Thus, for each probe type (positive and negative, forgiveness), each participant has a cosine for each experimental condition
\end{abstract} (compassionate reappraisal, emotion suppression, and offense rumination).

${ }^{2}$ To serve as a ground, we attached skin conductance pre-gelled Biopac EL507 snap electrodes fitted to LEAD110A electrode leads placed on the index and middle fingers of the left hand, after it had been rinsed with tap water. Data were sampled at 62.5 $\mathrm{HZ}$ and amplified by a Biopac GSR100C electrodermal response amplifier set for a gain of 5 mho/V. We followed methods for calculating change in GSR from pretrial baseline to imagery periods (subtracting the final 10s epoch value from each imagery epoch value, and then averaging all imagery epoch change scores). GSR reactivity scores were negative for all experimental imagery conditions, which we interpreted as habituation from pretrial baseline periods. Although both coping strategies produced lower GSR values compared to rumination, all ANOVAs for reappraisal versus rumination, suppression versus rumination, and

| the reappraisal effect versus the suppression effect were nonsignificant, all $F \mathrm{~s} \leq 2.34$, all $p \mathrm{~s} \geq .13$, all partial $\eta^{2} \mathrm{~s} \leq .04$.

Facial electromyography activity (EMG) was measured (Biopac EMG $100 \mathrm{C}$ units) on a second-to-second basis for the zygomaticus (cheek) muscle, orbicularis oculi (under eye) muscle, and corrugator supercilii (brow) muscle regions using two 4 mm EL258RT Biopac Ag-AgCl electrodes placed at each site on the left side of the face. Skin was first prepared with an alcohol pad and Biopac Gel 100. Each electrode was fitted with a Biopac ADD204 adhesive collar and filled with gel. EMG was sampled at $2000 \mathrm{~Hz}$ amplified by Biopac EMG100C amplifiers set for a gain of 1000 and using $10 \mathrm{~Hz}$ high-pass and $5 \mathrm{kHz}$ low-pass filters. EMG data was first digitally filtered using the Comb Band Stop Filter to select the line frequency at $60 \mathrm{~Hz}$ and overharmonics selecting all up to the Nyquist frequency. Data were filtered using the FIR Bandpass option to select the Bartlett window with a low frequency cutoff fixed at 28, high frequency cutoff fixed at 500, and Q coefficients set to 286. Next the EMG data were rectified and integrated by averaging over 10 samples and taking the root mean square of the entire wave form.

Electrocardiogram (ECG) data were measured by placing Biopac pre-gelled El503 snap electrodes fitted to Lead110S on the left rib and on the right clavicle. Rubbing alcohol was used to clean each electrode placement site. Data were sampled at $1000 \mathrm{~Hz}$ and amplified by $1000 \mathrm{~Hz}$ using the Biopac ECG100C electrocardiogram amplifier. Continuous R-R intervals were calculated in seconds.

${ }^{3}$ The Heart Rate Variability Specialized Analysis function of Acqknowledge used methods and produced values that were not consistent with guidelines and expected ranges based on the Task Force of the European Society of Cardiology and the North American Society of Pacing and Electrophysiology (1996) paper. Using the Task Force (1996) paper, Paul DeYoung wrote software that followed the published specifications for calculating the High Frequency (HF) component of the power spectrum to determine the parasympathetic contribution to the cardiac cycle. The R-R data for each120-s period were interpolated with cubic splines and then 1024 uniformly spaced values were calculated. A Welch periodogram estimate of the Power Spectrum Density (PSD) was calculated from the Fast Fourier Transform of de-trended subintervals of the 120 -s period (7 segments with a $50 \%$ overlap). Each subinterval was multiplied by a Hamming window. Results with this method correlated highly with values produced using the Root Mean Squared Successive Differences (RMSSD) method ( $r \mathrm{~s} \geq .8$ ). We further cross validated a susbset of results with two other programs (HRV Analysis Software 1.1 from the Biomedical Signal Analysis Group, Department of Applied Physics, University of Kuopio, Finland; Mindware HRV 2.51). 


\section{References}

Aldao, A., Nolen-Hoeksema, S., \& Schweizer, S. (2010). Emotion-regulation strategies across psychopathology: A meta-analytic review. Clinical Psychology Review, 30, 217-237.

Altamirano, L. J., Miyake, A., \& Whitmer, A. J. (2010). When mental inflexibility facilitates executive control: Beneficial side effects of ruminative tendencies on goal maintenance. Psychological Science, 21, 1377-1382.

Augustine, A. A. \& Hemenover, S. H. (2009). On the relative effectiveness of affect regulation strategies: A meta-analysis. Cognition and Emotion, 23, 1181-1220.

Berry, J. W., Worthington, E. L. Jr., Wade, N. G., Witvliet, C. V. O., Kiefer, R. P. (2005). Forgiveness, moral identity, and perceived justice in crime victims and their supporters. Humboldt Journal of Social Relations, 29, 136-162.

Bono, G., McCullough, M. E., \& Root, L. M. (2008). Forgiveness, feeling connected to others, and well-being: Two longitudinal studies. Personality and Social Psychology Bulletin, 34, 182-195.

Butler, E. A., Egloff, B., Wilhelm, F. H., Smith, N. C., Erickson, E. A., \& Gross, J. A. (2003). The social consequences of expressive suppression. Emotion, 3, 48-67.

Ciarocco, N. J., Vohs, K. D., \& Baumeister, R. F. (2010). Some good news about rumination: Task-focused thinking after failure facilitates performance improvement. Journal of Social and Clinical Psychology, 29, 1057-1073.

Dalgleish, T., Yiend, J., Schweizer, S., \& Dunn, B. (2009). Ironic effects of emotion suppression when recounting distressing memories. Emotion, 9, 744-749. 
Dunn, B. D., Billotti, D., Murphy, V., \& Dalgleish, T. (2009). The consequences of effortful emotion regulation when processing distressing material: A comparison of suppression and acceptance. Behaviour Research and Therapy, 47, 761-773.

Enright, R. (2001). Forgiveness is a choice: A step-by-step process for resolving anger and restoring hope. Washington, DC: American Psychological Association.

Enright, R., \& Fitzgibbons, R. (2000). Helping clients forgive: An empirical guide for resolving anger and restoring hope. Washington, DC: American Psychological Association.

Friedberg, J. P., Suchday, S., \& Shelov, D. V. (2007). The impact of forgiveness on cardiovascular reactivity and recovery. International Journal of Psychophysiology, 65, 87-94.

Giese-Davis, J., \& Spiegel, D. (2003). Emotional expression and cancer progression. In R.J. Davidson, K.R. Scherer, \& H.H. Goldsmith (Eds.), Handbook of affective sciences (pp. 1053-1082. New York: Oxford University Press.

Gordon, K., Burton, S., \& Porter, L. (2004). Predicting the intentions of women in domestic violence shelters to return to partners: Does forgiveness play a role? Journal of Family Psychology, 18, 331-338.

Gross, J. J. (1998). Antecedent- and response-focused emotion regulation: Divergent consequences for experience, expression and physiology. Journal of Personality and Social Psychology, 74, 224-237.

Gross, J. J. (2007). Handbook of emotion-regulation. New York: Guilford Press.

Gross, J. J., \& John, O.P. (2003). Individual differences in two emotion regulation processes: Implications for affect, relationships, and well-being. Journal of Personality and Social Psychology, 85, 384-362. 
Gross, J. J. \& Levenson, R. W. (1993). Emotional suppression: Physiology, self-report, and expressive behavior. Journal of Personality and Social Psychology, 64, 970-986.

Gross, J. J. \& Levenson, R.W. (1997). Hiding feelings: The acute effects of inhibiting negative and positive emotion. Journal of Abnormal Psychology, 106, 95-103.

Hargrave, T. (2001). Forgiving the devil: Coming to terms with damaged relationships. Phoenix, AZ: Zeig, Tucker, and Theisen, Inc.

Kachadourian, L. K., Fincham, F., \& Davila, J. (2005). Attitudinal ambivalence, rumination, and forgiveness of partner transgressions in marriage. Personality and Social Psychology Bulletin, 31, 334-342.

Lawler, K. A., Younger, J. W., Piferi, R. L., Billington, E., Jobe, R., Edmondson, K., \& Jones, W.H. (2003). A change of heart: Cardiovascular correlates of forgiveness in response to interpersonal conflict. Journal of Behavioral Medicine, 26, 373-393.

Lawler-Row, K. A., Karremans, J. C., Scott, C., Edlis-Matityahou, M., \& Edwards, L. (2008). Forgiveness, physiological reactivity and health: The role of anger. International Journal of Psychophysiology, 68, 51-58.

Luchies, L. B., Finkel, E. J., McNulty, J. K., \& Kumashiro, M. (2010). The doormat effect: When forgiving erodes self-respect and self-concept clarity. Journal of Personality and Social Psychology, 98, 734-749.

Maio, G. R., Thomas, G., Fincham, F. D., Carnelley, K. B. (2008). Unraveling the role of forgiveness in family relationships. Journal of Personality and Social Psychology, 94, 307-319.

Mauss, I. B., Cook, C. L., Cheng, J. Y. J., \& Gross, J. J. (2007). Individual differences in cognitive reappraisal: Experiential and physiological responses to an anger 
provocation. International Journal of Psychophysiology, 66, 116-124.

McCullough, M. E., Bono, G., \& Root, L. M. (2007). Rumination, emotion, and forgiveness: Three longitudinal studies. Journal of Personality and Social Psychology, 92, 490-505.

McCullough, M., Worthington, E., \& Rachal, K. (1997). Interpersonal forgiving in close relationships. Journal of Personality and Social Psychology, 73(2), 321-336.

McNulty, J. K. (2010). Forgiveness increases the likelihood of subsequent partner transgressions in marriage. Journal of Family Psychology, 24(6), 787-790.

Nolen-Hoeksema, S., Wisco, B., \& Lyubomirsky, S. (2008). Rethinking rumination. Perspectives on Psychological Science, 3, 400-424.

Pennebaker, J. W., Booth, R. J., \& Francis, M. E. (2007). Linguistic Inquiry and Word Count: LIWC [Computer software]. Austin, TX: LIWC.net.

Ray, R. D., Wilhelm, F. H., \& Gross, J. J. (2008). All in the mind's eye? Anger rumination and reappraisal. Journal of Personality and Social Psychology, 94, 133-145.

Richards, J. M., \& Gross, J. J. (1999). Composure at any cost? The cognitive consequences of emotion suppression. Personality \& Social Psychology Bulletin, 25, 1033-1044.

Rottenberg, J. \& Gross, J. J. (2007). Emotion and emotion regulation: A map for psychotherapy researchers. Clinical Psychology: Science and Practice, 14, 323-328.

Ryff, C. D., \& Singer, B. H. (2003). The role of emotion on pathways to positive health. In R. J. Davidson, K. R. Scherer, \& H. H. Goldsmith (Eds.), Handbook of affective sciences (pp. 1083-1104). New York: Oxford University Press. 
Sandage, S. J., \& Jankowski, P. J. (2010). Forgiveness, spiritual instability, mental health symptoms, and well-being: Mediator effects of differentiation of self. Psychology of Religion and Spirituality, 2, 168-180.

Sandage, S. J., \& Worthington, E. L., Jr. (2010). Comparison of two group interventions to promote forgiveness: Empathy as a mediator of change. Journal of Mental Health Counseling, 32, 35-57.

Subkoviak, M., Enright, R., Wu, C., \& Gassin, E. (1995). Measuring interpersonal forgiveness in late adolescence and middle adulthood. Journal of Adolescence, 18(6), 641-655.

Task Force of the European Society of Cardiology and the North American Society of Pacing and Electrophysiology. (1996). Heart rate variability: Standards of measurement, physiological interpretation, and clinical use. European Heart Journal, 17, 354-381.

Thayer, J. F., \& Brosschot, J. F. (2005). Psychosomatics and psychopathology: Looking up and down from the brain. Psychoneuroendocrinology, 30, 1050-1058.

Waltman, M. A., Russell, D. C., Coyle, C. T., Enright, R. D., Holter, A. C., \& Swoboda, C. M. (2009). The effects of a forgiveness intervention on patients with coronary artery disease. Psychology and Health, 24, 11-27.

Watkins, E. (2008). Constructive and unconstructive repetitive thought. Psychological Bulletin, 134, 163-206.

Wegner, D., Erber, R., \& Zanakos, S. (1993). Ironic processes in the mental control of mood and mood-related thought. Journal of Personality and Social Psychology, 65(6), 1093-1104.

Witvliet, C. V. O., Knoll, R. W., Hinman, N. G., and DeYoung, P. A. (2010). Compassionfocused reappraisal, benefit-focused reappraisal, and rumination after an interpersonal 
offense: Emotion regulation implications for subjective emotion, linguistic responses, and physiology. The Journal of Positive Psychology, 5, 226-242.

Witvliet, C. V. O., Ludwig, T., \& Vander Laan, K. (2001). Granting forgiveness or harboring grudges: Implications for emotions, physiology, and health. Psychological Science, 12, 117-123.

Witvliet, C.V.O., \& McCullough, M.E. (2007). Forgiveness and health: A review and theoretical exploration of emotion pathways. In S. Post (Ed.), Altruism and Health: Perspectives from Empirical Research, pp. 259-276. Oxford University Press.

Witvliet, C. V. O., Worthington, E. L., Root, L. M., Sato, A. F., Ludwig, T. E., \& Exline, J. J. (2008). Retributive justice, restorative justice, and forgiveness: An experimental psychophysiology analysis. Journal of Experimental Social Psychology, 44, 10-25.

Witvliet, C. V. O. \& Vrana, S. R. (1995). Psychophysiological responses as indices of affective dimensions. Psychophysiology, 32, 436-443.

Worthington, E. L., Jr. (Ed.). (2005). Handbook of Forgiveness. New York: Brunner-Routledge.

Worthington, E. L., Jr. (2009). A just forgiveness: Responsible healing without excusing injustice. Downers Grove, IL: InterVarsity Press.

Worthington, E. L., Jr., \& Sotoohi, G. (2009). Physiological assessment of forgiveness, grudges, and revenge: Theories, research methods, and implications. In A.M. Columbus (Ed.), Advances in Psychology Research, volume 64. New York: Nova Science Publishers. 
Table 1. Mean, $F$-values, $d f$, and partial $\eta^{2}$ s for offense rumination and subsequent coping condition

Offense Rumination, Reappraisal, and Suppression 37

Offense (R) Reappraisal Offense (R) vs. Reappraisal

Offense (S) Suppression Offense (S) vs. Suppression

\begin{tabular}{|c|c|c|c|c|c|c|c|c|c|c|}
\hline Dependent Variable & $M$ & $M$ & $F$ & $(d f)$ & partial $\eta^{2}$ & $M$ & $M$ & $F$ & $(d f)$ & partial $\eta^{2}$ \\
\hline \multicolumn{11}{|c|}{ EMG (microVolts change from relevant Pretrial Baseline) } \\
\hline Zygomatic & 0.313 & 0.633 & $1.01^{\text {n.s. }}$ & 1,22 & .04 & 0.850 & 0.367 & $3.30^{\wedge}$ & 1,22 & .13 \\
\hline Corrugator & 0.678 & 0.431 & $0.80^{\text {n.s. }}$ & 1,43 & .02 & 0.764 & 0.202 & $4.16^{*}$ & 1,43 & .09 \\
\hline Orbicularis Oculi & 1.876 & 0.911 & $4.00 *$ & 1,44 & .08 & 2.713 & 0.769 & $5.67 *$ & 1,44 & .11 \\
\hline \multicolumn{11}{|c|}{ Cardiovascular measures (change from relevant Pretrial Baseline) } \\
\hline R-R interval average (ms) - & -11.857 & -1.460 & $2.52^{\text {n.s. }}$ & 1,45 & .05 & -10.547 & 10.730 & $9.85 * *$ & 1,43 & .19 \\
\hline $\operatorname{HF} \operatorname{HRV}\left(\mathrm{ms}^{2}\right)$ & 608.509 & -253.932 & $2.22^{\text {n.s. }}$ & 1,39 & .05 & -159.911 & -168.283 & $0.00^{\text {n.s. }}$ & 1,38 & .00 \\
\hline \multicolumn{11}{|l|}{ Ratings (1-7 range) } \\
\hline Negative-Positive Valence & 2.65 & 4.83 & $136.96 * * *$ & 1,51 & .73 & 2.96 & 4.10 & $28.90 * * *$ & 1,51 & .36 \\
\hline Empathy & 2.71 & 4.23 & $47.46^{* * *}$ & 1,51 & .48 & 2.69 & 3.27 & $6.97 *$ & 1,51 & .12 \\
\hline Decision to Forgive & 3.13 & 4.83 & $53.08 * * *$ & 1,51 & .51 & 3.42 & 3.73 & $2.48^{\text {n.s. }}$ & 1,51 & .05 \\
\hline Emotional Forgiveness & 2.61 & 4.51 & $75.30 * * *$ & 1,50 & .60 & 3.18 & 3.31 & $0.49^{\text {n.s. }}$ & 1,50 & .01 \\
\hline Control & 4.27 & 4.98 & $7.10 * *$ & 1,51 & .12 & 4.35 & 4.67 & $1.90^{\text {n.s. }}$ & 1,51 & .04 \\
\hline Anger & 4.15 & 2.90 & $19.21 * * *$ & 1,51 & .27 & 4.54 & 2.83 & $64.38^{* * *}$ & 1,51 & .56 \\
\hline Anxiety & 3.83 & 2.85 & $21.44^{* * *}$ & 1,51 & .30 & 3.92 & 3.06 & $15.06 * * *$ & 1,50 & .23 \\
\hline Sadness & 4.49 & 3.04 & $44.51 * * *$ & 1,50 & .47 & 4.54 & 3.29 & $24.13^{* * *}$ & 1,51 & .32 \\
\hline \multicolumn{11}{|c|}{ Latent Semantic Analysis (cosines of distance from probe type) } \\
\hline Negative Emotion Language & 0.32 & 0.28 & $4.88 *$ & 1,53 & .08 & 0.31 & 0.27 & $6.22 *$ & 1,53 & .11 \\
\hline Positive Emotion Language & 0.18 & 0.23 & $13.25^{* * *}$ & 1,53 & .20 & 0.19 & 0.18 & $1.01^{\mathrm{n} . \mathrm{s}}$ & 1,53 & .02 \\
\hline Forgiveness-Related Languag & ge 0.13 & 0.13 & $0.01^{\text {n.s. }}$ & 1,53 & .00 & 0.13 & 0.09 & $11.45^{* * *}$ & 1,53 & .18 \\
\hline \multicolumn{11}{|c|}{ Linguistic Inquiry and Word Count-LIWC2007 } \\
\hline Social Words & 9.24 & 12.85 & $13.02 * * *$ & 1,53 & .20 & 11.13 & 9.47 & $2.39^{\text {n.s. }}$ & 1,53 & .04 \\
\hline Positive Emotion Words & 1.98 & 7.53 & $49.56 * * *$ & 1,53 & .48 & 2.15 & 4.02 & $7.29 * *$ & 1,53 & .12 \\
\hline Negative Emotion Words & 8.72 & 3.51 & $42.58 * * *$ & 1,53 & .45 & 7.46 & 4.17 & $18.87 * * *$ & 1,53 & .26 \\
\hline Forgiveness Words & 0.12 & 1.58 & $24.14^{* * *}$ & 1,53 & .31 & 0.21 & 0.38 & $0.91^{\text {n.s. }}$ & 1,53 & .02 \\
\hline
\end{tabular}

Note. ${ }^{*} p \leq .05 .{ }^{* *} p \leq .01 .{ }^{* * *} p \leq .001 . \wedge p=.08$. 
Table 2. Mean changes, $F$-values, $d f$, and partial $\eta^{2}$ for the reappraisal effect versus the suppression effect

\begin{tabular}{|c|c|c|c|c|c|}
\hline \multirow{2}{*}{ Dependent Variable } & \multirow{2}{*}{$\frac{\text { Reappraisal Effect }}{M}$} & \multirow{2}{*}{$\frac{\text { Suppression Effect }}{M}$} & \multicolumn{3}{|c|}{ Reappraisal Effect vs. Suppression Effect } \\
\hline & & & $F$ & $(d f)$ & partial $\eta^{2}$ \\
\hline \multicolumn{6}{|c|}{ EMG (microVolts change from relevant Pretrial Baseline) } \\
\hline Zygomatic & 0.320 & -0.483 & $4.38^{*}$ & 1,22 & .17 \\
\hline Corrugator & -0.247 & -0.561 & $1.02^{\text {n.s. }}$ & 1,43 & .02 \\
\hline Orbicularis Oculi & -0.965 & -1.945 & $0.98^{\text {n.s. }}$ & 1,44 & .02 \\
\hline \multicolumn{6}{|c|}{ Cardiovascular measures (change from relevant Pretrial Baseline) } \\
\hline R-R interval average (ms) & 10.791 & 21.277 & $1.42^{\text {n.s. }}$ & 1,43 & .03 \\
\hline $\operatorname{HF} \operatorname{HRV}\left(\mathrm{ms}^{2}\right)$ & 350.056 & -3.371 & $0.81^{\text {n.s. }}$ & 1,38 & .02 \\
\hline \multicolumn{6}{|l|}{ Ratings (-6 to 6) } \\
\hline Negative-Positive Valence & 2.17 & 1.13 & $15.90^{* * *}$ & 1,51 & .24 \\
\hline Empathy & 1.52 & 0.58 & $9.62 * *$ & 1,51 & .16 \\
\hline Decisional Forgiveness & 1.69 & 0.31 & $24.18^{* * *}$ & 1,51 & .32 \\
\hline Emotional Forgiveness & 1.90 & 0.14 & $40.69 * * *$ & 1,50 & .45 \\
\hline Control & 0.71 & 0.33 & $1.17^{\text {n.s. }}$ & 1,51 & .02 \\
\hline Anger & -1.25 & -1.71 & $1.76^{\text {n.s. }}$ & 1,51 & .03 \\
\hline Anxiety & -1.02 & -0.86 & $0.34^{\text {n.s. }}$ & 1,50 & .01 \\
\hline Sadness & -1.45 & -1.24 & $0.44^{\text {n.s. }}$ & 1,50 & .01 \\
\hline \multicolumn{6}{|c|}{ Latent Semantic Analysis (Cosine Distance from Probe) } \\
\hline Negative Emotion Language & -0.03 & -0.03 & $0.00^{\text {n.s. }}$ & 1,53 & .00 \\
\hline Positive Emotion Language & 0.05 & -0.01 & $12.36 * * *$ & 1,53 & .19 \\
\hline Forgiveness-Related Languag & ge 0.00 & -0.04 & $5.06^{*}$ & 1,53 & .09 \\
\hline \multicolumn{6}{|l|}{ Linguistic Inquiry and Word Count } \\
\hline Social Words & 3.61 & -1.65 & $13.99 * * *$ & 1,53 & .21 \\
\hline Positive Emotion Words & 5.55 & 1.87 & $11.67 * * *$ & 1,53 & .18 \\
\hline Negative Emotion Words & -5.21 & -3.28 & $2.96^{\text {n.s. }}$ & 1,53 & .05 \\
\hline Forgiveness Words & 1.46 & 0.17 & $13.68^{* * *}$ & 1,53 & .21 \\
\hline
\end{tabular}

Note. ${ }^{*} p \leq .05 .{ }^{* *} p \leq .01 .^{* * *} p \leq .001$. 